\title{
Diverticulitis yeyunal con neumoperitoneo asintomático. Consideraciones del manejo no operatorio
}

\author{
Jejunal diverticulitis with asymptomatic pneumoperitoneum. \\ Non-operative management considerations
}

\author{
Samuel Limón Suárez, * Alejandro Lastiri López, * Juan Carlos Ramírez Núñez, \\ Víctor Raúl Carbajal Saldaña, ${ }^{\S}$ Oscar Alejandro Mora Torres ${ }^{\llbracket}$
}

Citar como: Limón SS, Lastiri LA, Ramírez NJC, Carbajal SVR, Mora TOA. Diverticulitis yeyunal con neumoperitoneo asintomático. Consideraciones del manejo no operatorio. Acta Med Grupo Angeles. 2021; 19 (3): 382-386. https://dx.doi.org/10.35366/101732

\section{Resumen}

Introducción: Los divertículos yeyunales son saculaciones localizadas en el borde mesentérico del yeyuno, cuya incidencia es de 0.2 a 4\%. El neumoperitoneo suele ocasionar abdomen agudo quirúrgico, pero puede presentarse en patologías que no requieren exploración quirúrgica. Objetivo: Reconocer esta patología como causa infrecuente de dolor abdominal crónico o abdomen agudo quirúrgico. Establecer recomendaciones para su manejo. Caso clínico: Hombre de 66 años con dolor abdominal intenso, tipo cólico, postprandial, nocturno con distensión abdominal severa, meteorismo, náusea persistente, diarrea crónica y saciedad temprana con pérdida del apetito. Abdomen doloroso, timpánico, peristalsis muy incrementada sin datos de irritación peritoneal. Antecedentes personales patológicos (APP): hipertensión arterial sistémica controlada con antagonistas del receptor de la angiotensina 2 (ARA-2). Se realiza tomografía axial computarizada (TAC) de abdomen con reporte de diverticulitis yeyunal y aire libre en cavidad abdominal. Conclusión: La diverticulitis yeyunal, patología infrecuente, es susceptible de tratamiento médico conservador siempre y cuando las condiciones del paciente lo permitan; en casos de complicaciones, el tratamiento quirúrgico con resección y anastomosis intestinal es el abordaje recomendado.

Palabras clave: Diverticulitis yeyunal, neumoperitoneo asintomático, tratamiento diverticulitis yeyunal.

\section{Abstract}

Introduction: Jejunal diverticula are saculations located on the mesenteric edge of the jejunum whose incidence is 0.2 to $4 \%$. Pneumoperitoneum frequently translates acute surgical abdomen but can occur in pathologies that do not require surgical exploration. Objective: To recognize this pathology as an infrequent cause of chronic abdominal pain or acute surgical abdomen. Establish recommendations for its management. Clinical case: 66 years old male with intense colic, postprandial, nocturnal abdominal pain with severe abdominal distension, meteorism, persistent nausea, chronic diarrhea and early satiety with loss of appetite. Abdomen painful, tympanic, peristalsis greatly increased without data of peritoneal irritation. APP: systemic arterial hypertension controlled with ARA-2. A CT scan of the abdomen is performed with a report of jejunal diverticulitis and open air in the abdominal cavity. Conclusion: Jejunal diverticulitis, infrequent pathology, is susceptible to conservative medical treatment as long as the patient's conditions allow; in cases of complications, surgical treatment with resection and intestinal anastomosis is the recommended approach.

Keywords: Jejunal diverticulitis, asymptomatic pneumoperitoneum, jejunal diverticulitis treatment.

* Cirugía General y Laparoscópica Avanzada, Hospital Ángeles Metropolitano. México.

* Cirugía General y Laparoscópica Avanzada. Hospital General de Cholula, Secretaría de Salud. México.

$\S$ Radiólogo, Departamento de Radiología e Imagen del Hospital Ángeles Metropolitano. México.

" Médico residente de tercer año de Cirugía General del Hospital Ángeles Lomas, Universidad Nacional Autónoma de México. México.

Correspondencia:

Dr. Samuel Limón Suárez

Correo electrónico: limonsamuel@gmail.com

Aceptado: 29-09-2020.

www.medigraphic.com/actamedica 


\section{INTRODUCCIÓN}

La diverticulosis yeyunal es una entidad rara, su prevalencia es aproximadamente de $1-2 \%$ variando de $0.02-0.4 \%$ en estudios de contraste del tubo digestivo, llegando hasta $4.0 \%$ en necropsias. ${ }^{1}$ Su incidencia aumenta con la edad, con un pico en la sexta y séptima décadas de la vida. ${ }^{2}$ Los primeros reportes de divertículos de yeyuno fueron hechos por Sommervit en 1794, Voigtel en 1804 y Sir Astley Cooper en 1807; los primeros en reportar las complicaciones derivadas de esta enfermedad fueron Virchow en 1853 y Osler en 1881. Gordinier y Sanpson en 1906 describieron el primer paciente que se sometió a cirugía por diverticulosis de yeyuno. En 1920, se reportaron los primeros cinco casos diagnosticados por radiología, y un año después Hunt y Cook resecaron exitosamente un divertículo de yeyuno. ${ }^{2}$

En lo que respecta al neumoperitoneo, la causa más frecuente es iatrogénica posterior a cirugía abdominal, es un hallazgo con frecuencia radiológico que en la mayor parte de contextos clínicos conduce a ruptura de víscera hueca y que requiere intervención quirúrgica urgente; ${ }^{3} \sin$ embargo, en ausencia de hallazgos clínicos de irritación peritoneal, debe hacer sospechar causas "espontáneas" de neumoperitoneo, las cuales con frecuencia no requieren intervención quirúrgica. ${ }^{4}$

La etiología de los divertículos yeyunales no es clara, aunque existen múltiples teorías, como la que explica que la formación de los divertículos puede estar dada por disfunción o discinesia del músculo liso del yeyuno, que desencadenan una alteración en la contracción. En su estudio con manometrías de yeyuno, Kongara y colaboradores reportan que en el periodo posterior a la ingestión de alimentos existen unas contracciones de mayor amplitud y duración que las normales; este trastorno se puede presentar de forma simultánea, lo cual conlleva aumento de la presión en la luz del intestino y predisposición a la formación de los divertículos. ${ }^{5}$

La sintomatología de la diverticulitis yeyunal se presenta en 10 a $19 \%$ de los pacientes, los cuales pueden presentar cuadros de dolor abdominal agudo con complicaciones como sangrado, perforación, intususcepción, vólvulo intestinal, oclusión intestinal, fístula y sepsis abdominal o en su mayoría (60\%) con cuadros crónicos como náusea y vómito ocasional, distensión y dolor abdominal, pérdida de peso, malabsorción, suboclusión y cuadros de diarrea alternante, los cuales conducen con frecuencia a diagnósticos y tratamientos errados. ${ }^{6}$

La tomografía abdominal con contraste intravenoso y oral es la herramienta de diagnóstico de elección, incluso si no se identifican todos los divertículos del intestino delgado, puede mostrar una lesión en masa que contiene burbujas de aire extraluminales, asas de intestino delgado dilatadas con reforzamiento del contraste intravenoso, pared engrosada, así como la participación del tejido circundante (p. ej: fístula entre el intestino delgado, colon y vejiga) y el aspecto hiperdenso inflamatorio del mesenterio.

El tratamiento de esta patología depende de la situación clínica del paciente, en casos de complicaciones o presencia de abdomen agudo, la cirugía con resección del segmento afectado y anastomosis será el objetivo por seguir. En casos de sintomatología sin urgencia quirúrgica o de neumoperitoneo asintomático, el reposo intestinal y terapia antimicrobiana puede ser una opción válida. ${ }^{8}$

\section{CASO CLÍNICO}

Hombre de 66 años valorado en consulta externa de Hospital Ángeles Metropolitano con un cuadro de más de cinco años de evolución que se agudizó los últimos 10 días, caracterizado por dolor abdominal general tipo cólico de predominio postprandial y nocturno asociado a distensión abdominal severa y meteorismo. En las últimas 24 horas se agrega estado nauseoso persistente y saciedad temprana con pérdida del apetito. Refiere episodios de evacuaciones diarreicas alternadas con estreñimiento. Como antecedente de importancia, fue multitratado de forma crónica con antiespasmódicos y antimicrobianos en institución de asistencia pública como síndrome de intestino irritable y cirrosis hepática alcohólica.

Antecedentes personales patológicos de importancia para padecimiento actual: hipertensión arterial sistémica

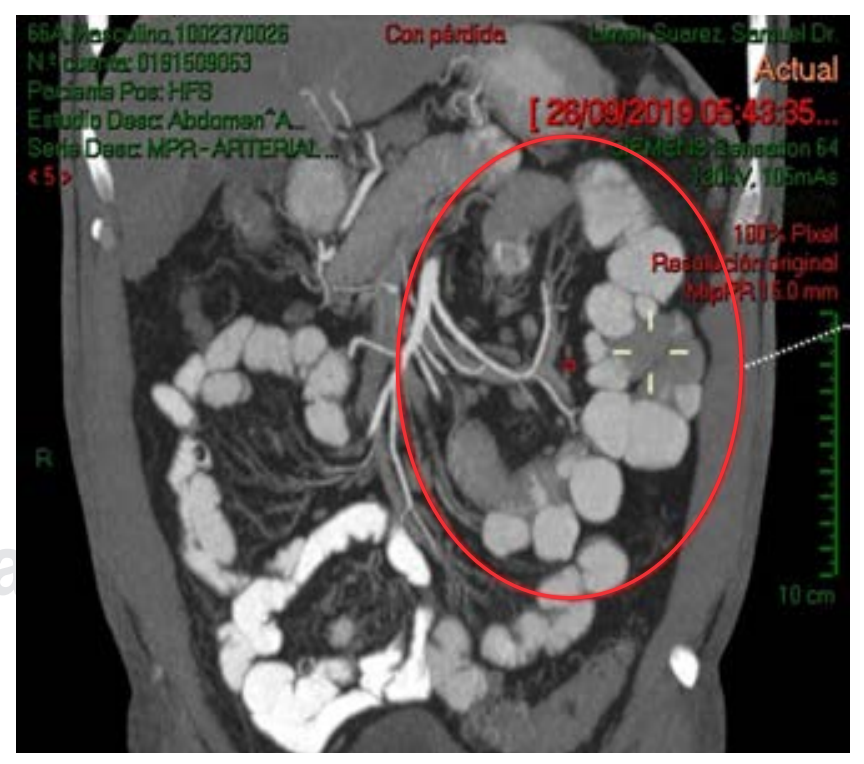

Figura 1: Divertículos en yeyuno terminal, se observa el realce por el contraste oral y la densidad diferente al contenido intraluminal. 


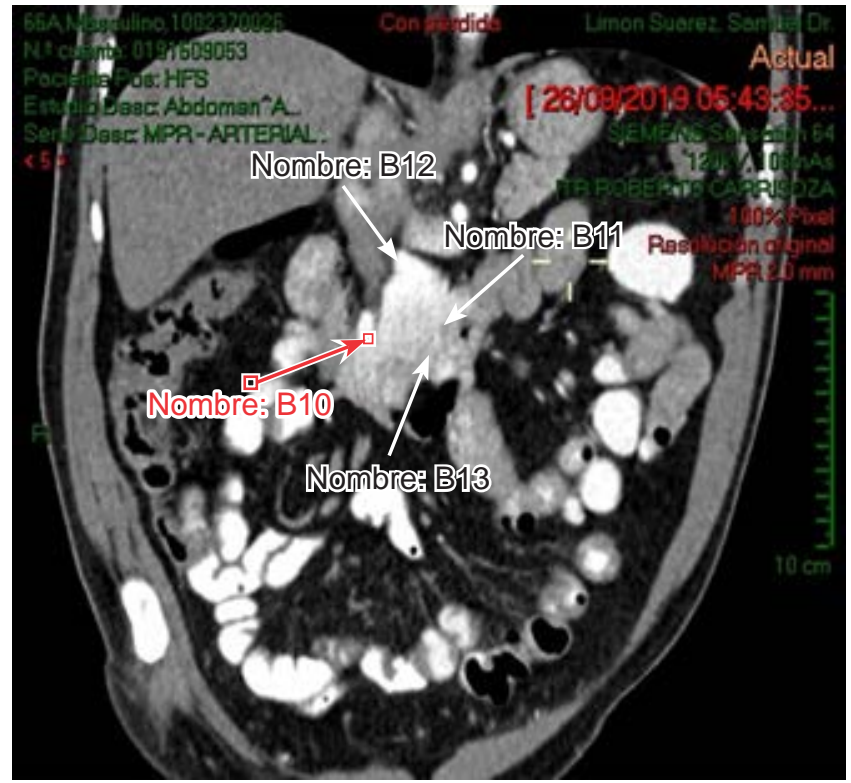

Figura 2: Se observa en yeyuno proximal divertículo grande (flechas blancas) con probable sitio de microperforación y ligera extravasación de material de contraste (flecha roja). Sin observar plastrones, colecciones periyeyunales o colecciones a distancia.

de larga evolución tratada con losartán de 50 mg cada 24 horas, refiere tabaquismo, tres cigarrillos al día durante los últimos 30 años (índice tabáquico [IT] 4.5). Quirúrgicos: apendicetomía abordaje abierto en la infancia y circuncisión hace dos meses.

Exploración física: presión arterial de 130/80, frecuencia cardiaca de 70, frecuencia respiratoria de 18 con saturación de oxígeno al aire ambiente de 93\% y temperatura de $36.5{ }^{\circ} \mathrm{C}$. Cabeza y cuello sin alteraciones, cardiopulmonar sin compromiso agudo al momento. Abdomen distendido, timpánico generalizado con peristalsis muy incrementada sin ruidos metálicos, a la palpación superficial sin hiperalgesia o hiperbaralgesia abdominal, no se encuentran datos francos de irritación peritoneal; a la palpación profunda, muy doloroso en mesogastrio e hipocondrio izquierdo, no se palpan visceromegalias o tumoraciones; sin más datos patológicos que agregar.

Ante los hallazgos, se inicia manejo sintomático con antiespasmódico y solicitamos tomografía axial computarizada (TAC) abdominal con contraste intravenoso y oral con reporte de abundante gas libre en cavidad abdominal, múltiples divertículos yeyunales desde $5 \mathrm{~mm}$ hasta $2 \mathrm{~cm}$, los mayores con sitio de probable perforación intestinal en topografía de yeyuno distal hacia mesogastrio y fosa iliaca izquierda. No hay líquido libre abdominal ni se observan plastrones. Se integra el diagnóstico de diverticulitis yeyunal y neumoperitoneo (Figuras 1 a 3).
Se decide iniciar tratamiento médico y manejo conservador en respuesta a los hallazgos tomográficos ya descritos, puesto que no existían datos de abdomen agudo quirúrgico, de respuesta inflamatoria sistémica o sepsis abdominal que justificaran la intervención quirúrgica; se prescriben 10 días de antimicrobiano con cefalosporina de tercera generación y metronidazol, antiespasmódico, inhibidor de bomba de protones y medidas higiénico-dietéticas con remisión completa de sintomatología.

\section{DISCUSIÓN}

A diferencia de los divertículos colónicos, la historia natural de los divertículos de intestino delgado no está bien estudiada, tienen una incidencia de $3 \%$ con una mediana de edad de aparición de 75 años, siendo la gran mayoría asintomáticos, pero pudiendo debutar con cuadros de abdomen agudo quirúrgico o con cuadros crónicos donde el punto cardinal es el dolor abdominal recurrente, acompañado de sintomatología abdominal poco específica como náusea o cambios en hábitos intestinales; su diagnóstico clínico es poco probable, son detectados en estudios de imagen o intraoperatorios. ${ }^{9}$ En la mayor parte de los casos en la literatura se encuentra reportado su manejo quirúrgico y anastomosis. ${ }^{10}$ Existen reportes de series pequeñas

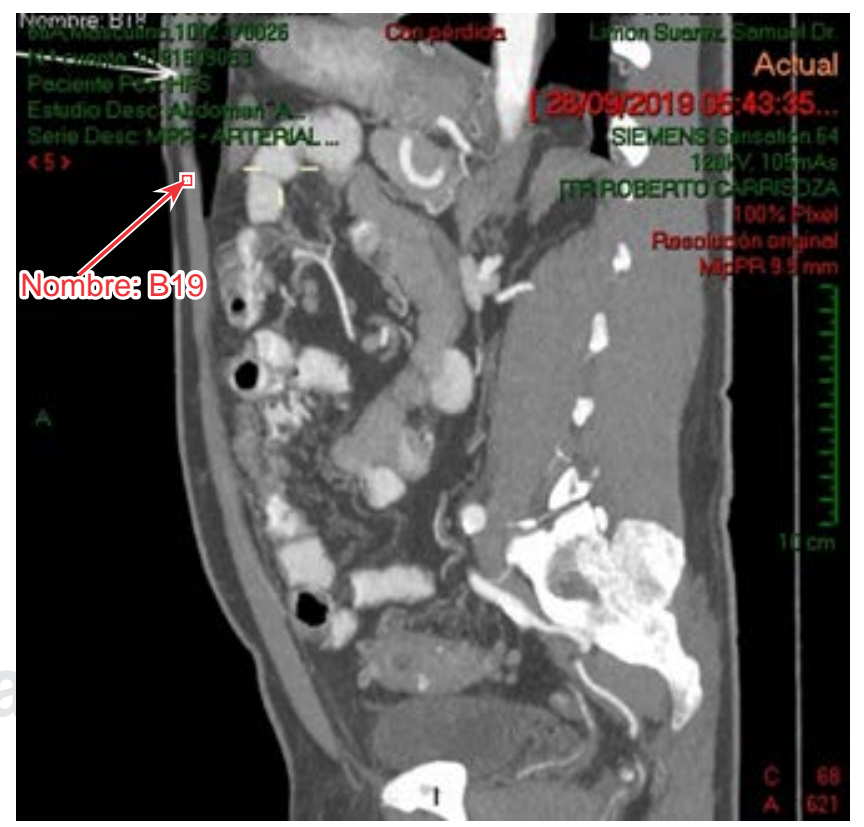

Figura 3: Tomografía axial computarizada de abdomen corte sagital. Se identifica hacia cefálico abundante aire libre en cavidad abdominal en todo el espacio subdiafragmático derecho e izquierdo (flecha roja). 
(cuatro casos) donde la mitad de los pacientes presentaron complicaciones como hemorragia y obstrucción, pero todos fueron sometidos a manejo quirúrgico con buenos resultados; ${ }^{11}$ también hay reportes de casos donde autores como Novak y colaboradores y Colvin y su equipo de investigadores describen buenos resultados sólo con terapia antimicrobiana y descompresión intestinal. ${ }^{12,13}$ Asimismo, en una revisión retrospectiva de 208 pacientes, Akhrass y su grupo describen sólo dos casos manejados de manera conservadora, obteniendo resultados curativos en un paciente, mientras el otro fue sometido a manejo quirúrgico. De igual manera, encontraron que hasta $46 \%$ de los casos tenían complicaciones al momento del diagnóstico como sangrado, perforación u obstrucción. ${ }^{14}$

Aunque no hay un consenso establecido en la literatura médica para su clasificación o manejo, autores como Lebert y colaboradores propusieron disponer los casos acorde a la clasificación de Hinchey modificada para diverticulitis colónica sin obtener una buena correlación. ${ }^{15}$ Por otro lado, Zi Qin y su equipo describieron un algoritmo que divide a los pacientes en tres grandes grupos basados en hallazgos tomográficos: diverticulitis no complicada o grado 1 , que corresponde a inflamación periyeyunal o flemón; diverticulitis complicada o grado 2 subdividida en perforación contenida (2A) o perforación libre con peritonitis (2B); y diverticulitis severamente complicada o grado 3, que corresponde a los casos que al momento del diagnóstico tienen sangrado y obstrucción. La aparición de neumoperitoneo al momento del diagnóstico tomográfico no necesariamente equivale a perforación libre, puesto que pueden existir microperforaciones a través de la membrana intestinal semipermeable con fuga de una considerable cantidad de gas a la cavidad peritoneal. Basados en esta clasificación, proponen que todos los pacientes grado 1 y $2 \mathrm{~A}$ deben ser tratados en un inicio con manejo antimicrobiano y reposo intestinal, monitorizando a los pacientes en busca de deterioro clínico o incremento en los reactantes de la inflamación durante al menos 72 horas antes de decidir continuar con el manejo médico conservador o manejo quirúrgico definitivo. En cuanto a los pacientes grado 2B y 3 , el abordaje inicial recomendado es la resección intestinal con entero-entero anastomosis con abordaje abierto o laparoscópico dependiendo de la experiencia del grupo quirúrgico, desestimando sutura primaria en el defecto o diverticulectomía por el alto índice de mortalidad que conlleva. En el caso de pacientes con oclusión intestinal (grado 3) causada por microperforación localizada o proceso inflamatorio severo, se puede intentar en un inicio manejo conservador, siempre y cuando las condiciones clínicas lo permitan. ${ }^{16}$

En cuanto a casos con sangrado activo (grado 3), la resucitación hidroelectrolítica, manejo de hemoderivados y la localización y resección sólo del segmento donde hay sangrado en caso de diverticulosis extensa o de todo el segmento afectado en caso de diverticulosis localizada constituyen el abordaje recomendado; sin embargo, ante la dificultad técnica que implica localizar el sitio exacto de sangrado en un segmento largo de intestino delgado con divertículos, y en el contexto de un paciente sin inestabilidad hemodinámica severa, la angiografía mesentérica y embolización son una opción válida. Aunque el éxito de este procedimiento para patología de intestino delgado sigue siendo menor que para patología colónica, en los últimos años se ha visto una mejoría notable en las tasas de éxito de este procedimiento. ${ }^{17}$ García-Granero y colaboradores identificaron tres criterios para manejo conservador de esta patología: 1) no existencia de irritación peritoneal; 2) estabilidad hemodinámica; y 3) tomografía con aire libre en cavidad, pero sin presencia de absceso localizado o a distancia. Ellos proponen revaloración a las 48 horas para decidir si no hay mejoría clínica y llevar al paciente a resección intestinal. ${ }^{18}$

En nuestro caso en particular, a pesar del hallazgo inicial ominoso de aire libre en cavidad abdominal, que en un contexto clínico diferente equivale a exploración quirúrgica mandatoria, se tomó en cuenta el buen estado general del paciente, la ausencia de respuesta inflamatoria sistémica, irritación peritoneal o sepsis para decidir iniciar un manejo conservador. Con base en los hallazgos tomográficos y clínicos, el paciente quedó clasificado como diverticulitis yeyunal grado 2A, cumplía con los tres criterios de GarcíaGranero para iniciar manejo conservador a base de antimicrobiano y reposo intestinal, por lo que, a pesar del hallazgo de aire libre en cavidad abdominal, decidimos iniciar doble esquema ambulatorio con ceftriaxona-metronidazol, reposo intestinal de sólidos por 48 horas y revaloración en búsqueda intencionada de aparición de datos de irritación peritoneal o respuesta inflamatoria sistémica. A las 48 horas se encuentra al paciente con una mejoría notable de la sintomatología, sin datos de abdomen agudo, afebril, precordio normorrítmico y normodinámico con adecuada saturación de oxígeno al aire ambiente, por lo que se inicia dieta blanda y se le indica completar 10 días de esquema antimicrobiano, obteniendo una resolución completa de la sintomatología a la revisión en la consulta externa 15 días después de iniciado el tratamiento.

\section{CONCLUSIÓN}

Basados en la literatura existente, aún no hay un manejo estandarizado y definitivo para estos pacientes. Merecen una mención aparte aquellos casos con diverticulitis yeyunal que se presentan con neumoperitoneo, pero sin datos de síndrome de respuesta inflamatoria sistémica (SRIS) o 
abdomen agudo, donde la falta de pericia clínica puede conducir a laparotomías innecesarias e incremento de la morbilidad postoperatoria. ${ }^{19}$ Por lo anterior, las clasificaciones de Zi Quin y colaboradores y los criterios de GarcíaGranero pueden ser de extrema utilidad para orientar en el manejo inicial de estos pacientes, aunque aún no son clasificaciones y algoritmos estandarizados puesto que son dependientes de la correcta interpretación de los hallazgos tomográficos y no toman en cuenta ciertos aspectos, como tamaño de abscesos intraabdominales, para clasificar la patología ni se establecen en los algoritmos criterios para manejo ambulatorio u hospitalización mandatoria; pueden ser de mucha utilidad en asociación con un adecuado contexto clínico. Todo paciente con diverticulitis yeyunal sin datos de respuesta inflamatoria sistémica, abdomen agudo quirúrgico, sangrado u oclusión intestinal, aun con presencia de aire libre en cavidad abdominal, debe ser considerado para manejo conservador inicial. Dependerá del criterio clínico del cirujano, la edad y presencia o ausencia de comorbilidades del paciente para que se decida si es candidato a tratamiento ambulatorio u hospitalización por el alto riesgo de falla del manejo médico, deterioro clínico y subsecuente necesidad de resolución quirúrgica. Con una persona hospitalizada para manejo conservador, es mandatoria la vigilancia clínica, determinación frecuente de reactantes inflamatorios y la revaloración tomográfica a las 48 o 72 horas máximo para determinar si es necesaria la intervención quirúrgica. Por otro lado, cuando al momento del diagnóstico se presenta un cuadro de abdomen agudo quirúrgico, respuesta inflamatoria sistémica persistente con sepsis abdominal, inestabilidad hemodinámica o sangrado masivo de tubo digestivo, la exploración quirúrgica mediante laparotomía para resección del segmento afectado y la anastomosis conforman una conducta a seguir. El abordaje laparoscópico no se desestima teniendo en cuenta dos factores clave: la pericia del grupo quirúrgico, que pudiera influir en el tiempo operatorio, y las condiciones clínicas del paciente al momento de decidir la intervención. No se recomienda cierre primario de defecto o diverticulectomía por el alto índice de morbimortalidad que conllevan.

\section{REFERENCIAS}

1. Noer T. Non-Meckelian diverticula of the small bowel. The incidence in an autopsy material. Acta Chir Scand. 1960; 120: 175-179.
2. Woods K, Williams E, Melvin W, Sharp K. Acquired jejunoileal diverticulosis and its complications: a review of the literature. Am Surg. 2008; 74 (9): 849-854.

3. Winek TG, Mosely HS, Grout G, Luallin D. Pneumoperitoneum and its association with ruptured abdominal viscus. Arch Surg. 1988; 123 (6): 709-712.

4. Madura MJ, Craig RM, Shields TW. Unusual causes of spontaneous pneumoperitoneum. Surg Gynecol Obstet. 1982; 154 (3): 417-420.

5. Kongara KR, Soffer EE. Intestinal motility in small bowel diverticulosis: a case report and review of the literature. J Clin Gastroenterol. 2000; 30 (1): 84-86.

6. Balducci G, Dente M, Cosenza G, Mercantini P, Salvi PF. Multiple giant diverticula of the foregut causing upper gastrointestinal obstruction. World J Gastroenterol. 2008; 14 (20): 3259-3261.

7. Macari M, Faust M, Liang H, Pachter HL. CT of jejunal diverticulitis: imaging findings, differential diagnosis, and clinical management. Clin Radiol. 2007; 62 (1): 73-77.

8. Prost A La Denise J, Douard R, Berger A, Cugnenc PH. Small bowel diverticulosis complicated by perforated jejunal diverticula: conservative and/or surgical management? Hepatogastroenterology. 2008; 55 (86-87): 1657-1659.

9. El-Haddawi F, Civil ID. Acquired jejuno-ileal diverticular disease: a diagnostic and management challenge. ANZJ Surg. 2003; 73 (8): 584-589.

10. Longo WE, Vernava AM 3rd. Clinical implications of jejunoileal diverticular disease. Dis Colon Rectum. 1992; 35 (4): 381-388.

11. Patel VA, Jefferis H, Spiegelberg B, Iqbal Q, Prabhudesai A, Harris S. Jejunal diverticulosis is not always a silent spectator: a report of 4 cases and review of the literature. World J Gastroenterol. 2008; 14 (38): 5916-5919.

12. Colvin HS, Kuenfoo C, Rajab TK, Sayadatas T. Non-surgical management of recurrent perforation of a jejunal diverticulum following previous segmental bowel resection: a case report. J Med Case Reports. 2009; 3: 7318.

13. Novak JS, Tobias J, Barkin JS. Nonsurgical management of acute jejunal diverticulitis: a review. Am J Gastroenterol. 1997; 92 (10): 1929-1931.

14. Akhrass R, Yaffe MB, Fischer C, Ponsky J, Shuck JM. Small-bowel diverticulosis: perceptions and reality. J Am Coll Surg. 1997; 184 (4): 383-388.

15. Lebert P, Millet I, Ernst O, Boulay-Coletta I, Corno L, Taourel P et al. Acute jejunoileal diverticulitis: multicenter descriptive study of 33 patients. AJR Am J Roentgenol. 2018; 210 (6): 1245-1251.

16. Ng ZQ, Theophilus M, Navadgi S, Menon T, Wijesuriya R. Jejunal diverticulitis: a single-center experience and proposed management algorithm. Surg Infect (Larchmt). 2019; 20 (6): 499-503. doi: 10.1089/ sur.2019.070.

17. Tan KK, Wong D, Sim R. Superselective embolization for lower gastrointestinal hemorrhage: an institutional review over 7 years. World J Surg. 2008; 32 (12): 2707-2715.

18. García-Granero A, Argüelles BG, Gallardo CM, Carreño O, Giner F, Pellino G et al. Short article: outcome of medical and surgical management for perforated jejunal diverticulitis. Eur J Gastroenterol Hepatol. 2019; 31 (1): 135-139.

19. Mularski RA, Sippel JM, Osborne ML. Pneumoperitoneum: a review of nonsurgical causes. Crit Care Med. 2000; 28 (7): 2638-2644.

Conflicto de intereses: No se declara conflicto de intereses en este artículo. 